\title{
Endoscopic microscopic transpedicular thoracic discectomy
}

\section{Technical note}

\section{Hae-Dong Jho, M.D., Ph.D.}

Department of Neurological Surgery, University of Pittsburgh School of Medicine, Pittsburgh, Pennsylvania

In an effort to make thoracic discectomy simple and less invasive while using direct visualization, a 70 š-angled lens endoscope has been adopted to visualize the ventral aspect of the spinal cord dura mater during microsurgical thoracic discectomy via a transpedicular approach. The patient is positioned in a 60 š forwardly inclined lateral position with the side of the lesion facing upward. After radiographic corroboration of the correct level, a transpedicular approach is made using a 1.5-cm-diameter tubular retractor through a 2-cm-long paramedian transverse skin incision. With the aid of an operating microscope, the ipsilateral facet joint, including the upper portion of the pedicle, is removed using a high-speed drill, thus exposing the neural foramen, intervertebral disc, and upper portion of the pedicle leading to the vertebral bodies. When the herniated disc and bone spur have been removed laterally in relation to the spinal cord, creating a cavity under the operating microscope, a 4-mm-diameter rigid endoscope with a 70š-angled lens is mounted to an endoscope holder so that the ventral aspect of the spinal cord dura mater can be visualized directly. With the aid of direct endoscopic visualization, the disc and bone spur, which compress the spinal cord anteriorly, are pushed away toward a cavity created at the intervertebral space and are removed using a downward-biting long-armed curette. Patients with myelopathy are kept overnight in the hospital; however, those with radiculopathy are discharged home on the same day as their operation. The surgical technique and two illustrative cases are reported.

Key Words * endoscopy * intervertebral disc displacement * minimally invasive neurosurgery * spine * thoracic spine * vertebra

Thoracic disc herniation has been surgically treated using thoracotomy, posterolateral extracavitary, costotransversectomy, and transpedicular approaches.[2-7] These surgical approaches have achieved comparable surgical outcomes, avoiding the catastrophic spinal cord damage that commonly occurred using a laminectomy approach.[1] Despite the avoidance of spinal cord damage, these surgical procedures are relatively invasive compared with cervical or lumbar discectomy. Recently, an endoscopic transthoracic discectomy was developed.[7] That endoscopic technique still requires three to four separate skin incisions for entry sites to the thoracic cavity, followed by thoracotomy tube drainage for a few days postoperatively. 
Among the different surgical techniques for thoracic discectomy, a transpedicular approach is the least invasive. [5,8] Although good surgical outcomes have been reported using the transpedicular approach, it does not provide direct visualization of the spinal cord ventrally while the surgeon is decompressing the spinal cord. In an attempt to make thoracic discectomy simple and easy while achieving comparable invasiveness to cervical or lumbar discectomy under direct visualization, a 70š-angled lens endoscope was adopted to visualize the anterior aspect of the spinal cord dura mater directly during microsurgical thoracic discectomy via a transpedicular approach.

This technique has been used in eight patients with thoracic disc herniation. The surgical outcome has been comparable to that of cervical or lumbar discectomy. The new technique has been performed on an outpatient basis for radiculopathy. In this article, the surgical technique is described and two illustrative cases are reported.

\section{OPERATIVE TECHNIQUE}

General anesthesia is induced in the patient using endotracheal intubation. The patient is positioned in a 60 š forwardly inclined lateral position with the side of the lesion facing upward (Fig. 1 upper left). An axillary roll is placed under the dependent axilla to prevent injury to the brachial plexus and shoulder joint. The hips and knees are gently flexed and a pillow is placed between the knees. The patient's position is secured with multiple tapes. An 18-gauge spinal needle is inserted $8 \mathrm{~cm}$ lateral from the midline and is directed toward the base of the spinous process for roentgenographic verification of the correct level. Once the correct level of disc herniation is confirmed, the spinal needle is withdrawn. Preparation and draping is done following aseptic technique. The surgical incisional line is marked in reference to the needle entry site and its directional angle. 


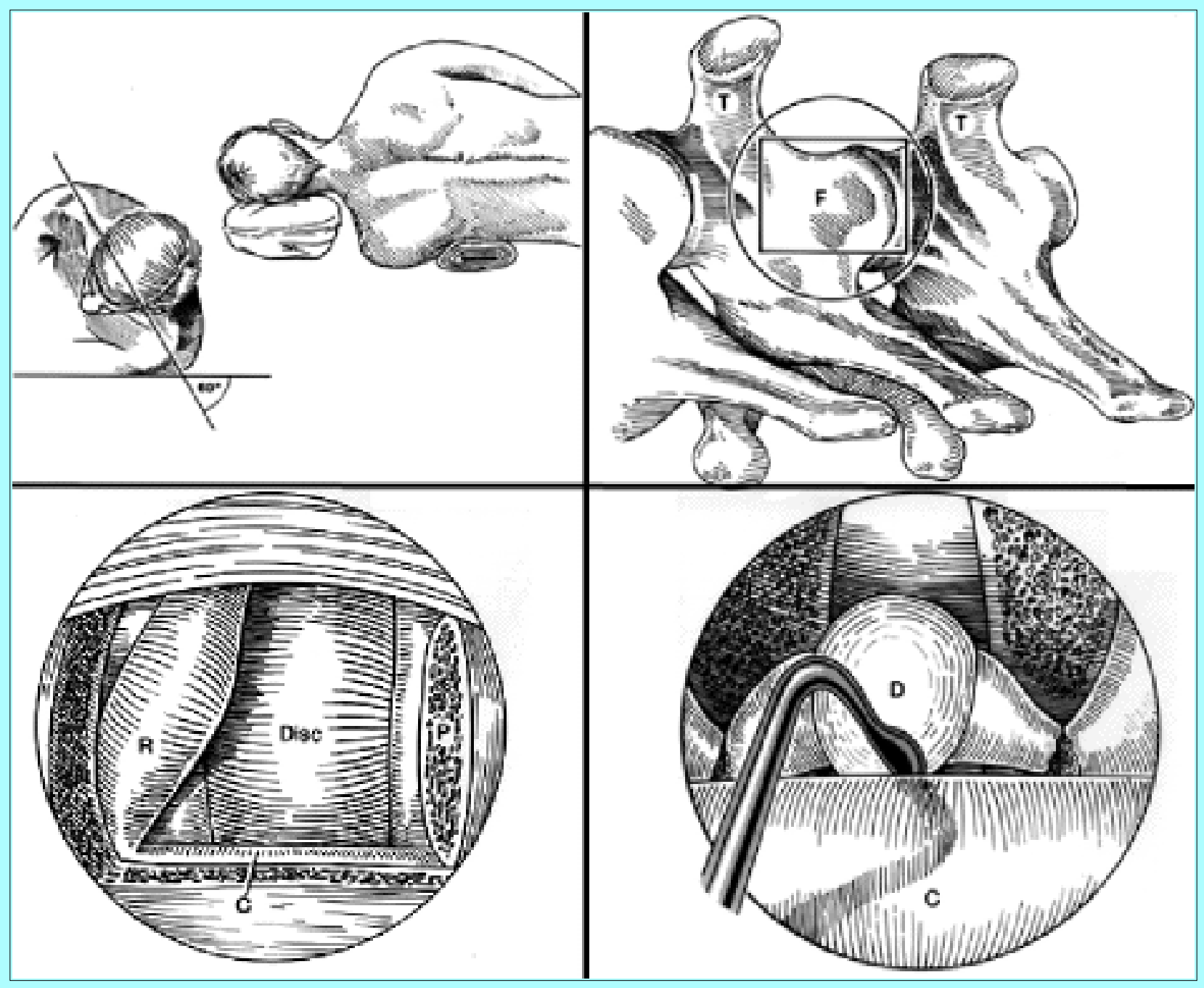

Fig. 1. Schematic drawings. Upper Left: Placement of the patient in a 60 š forwardly inclined lateral position. This positioning allows the surgeon to sit comfortably while operating using the operating microscope or endoscope. Upper Right: The circle surrounds the area to be exposed through a trocar retractor. The landmarks for the operating area are the transverse processes of the rostral and caudal vertebrae (T). The anatomical structure located medially and in between the transverse processes is the facet joint $(\mathrm{F})$. The area enclosed by the square is the facet joint and the upper portion of the pedicle to be removed using a high-speed drill. Lower Left: The facet joint and upper portion of the pedicle $(\mathrm{P})$ are removed using a high-speed drill with the aid of the operating microscope. When the yellow ligament is removed, the very lateral margin of the spinal cord dura mater $(\mathrm{C})$ and nerve root $(\mathrm{R})$ is exposed. Commonly the nerve root slides down along the inferior margin of the pedicle of the rostral vertebra; however, it is not uncommon to observe the nerve root emerging along the superolateral margin of the resected pedicle of the caudal vertebra. Lower Right: When bone spurs, inferior and superior to the intervertebral disc, are further removed using a high-speed drill and the lateral intervertebral disc is resected with the aid of the operating microscope, a cavity measuring approximately $1.5 \mathrm{~cm}$ is created lateral to the spinal cord. An underlying tunnel is then made medially with a downward-biting curette. This drawing demonstrates the endoscopic view of the remaining shell-like disc (D) and bone spur compressing the spinal cord $(\mathrm{C})$.

A 2-cm-long transverse skin incision is made, keeping the center of the incision approximately $3 \mathrm{~cm}$ 
lateral from the posterior midline. The subcutaneous tissue is divided along the incision line. A curvilinear fascial incision is made with a flap based at the midline. The paraspinal muscles are dissected from the spinous process, lamina, and transverse processes using a small periosteal elevator. A tubular trocar measuring $1.5 \mathrm{~cm}$ in diameter is introduced, exposing the facet and the lamina (Fig. 1 upper right). With the aid of an operating microscope, the facet, the lateral portion of the lamina, and the upper portion of the pedicle are removed using a high-speed drill. Because of the proximity of the upper portion of the pedicle to the intervertebral disc, 2 to $3 \mathrm{~mm}$ of the upper portion of the pedicle in the caudal vertebra need to be removed to provide adequate access to the spondylotic bone spur of the caudal vertebra. When the yellow ligament is removed, the very lateral margin of the spinal cord dura mater and the nerve root come into direct view (Fig. 1 lower left). The nerve root commonly slides down along the inferior margin of the pedicle of the rostral vertebra; however, it is not uncommon to find the nerve root just above the resected portion of the pedicle of the caudal vertebra as it emerges superolaterally in relation to the spinal cord. In cases of radiculopathy, the nerve root compression can be identified. Lateral bone spurs that are inferior and superior to the intervertebral disc are removed using a high-speed drill and the lateral intervertebral disc is also removed, creating a small cavity. The limited exposure of the spinal cord dura mater will not allow a surgeon to injure the spinal cord because the main portion of the spinal cord is still covered by the remaining ipsilateral lamina. Once the cavity, which measures approximately $1.5 \mathrm{~cm}$, is created at the lateral intervertebral disc space by resection of the bone spur and disc material, further resection of the bone can be performed medially with a downward-biting curette. This creates an underlying tunnel and leaves a shell at the posterior portion of the intervertebral disc and bone spur.

When this generous cavity is made lateral to the spinal cord and the aforementioned tunnel is made anterior to the spinal cord with the aid of an operating microscope, a 70š-angled lens endoscope can be applied to visualize the ventral aspect of the spinal cord dura mater directly (Fig. 1 lower right). An endoscope cleansing device, videocamera, monitor, and videoprinting equipment are assembled and connected to the endoscope. The endoscope is mounted to a custom-made endoscope holder. With the aid of direct endoscopic visualization, the underlying tunnel is further enlarged crossing the midline by removal of the bone spur and the intervertebral disc. The remaining shell-like herniated disc and bone spur, which are medially compressing the spinal cord, are then pushed away from the spinal cord toward the cavity using a custom-made long-armed downward-biting curette and removed using a curved suction cannula and pituitary rongeur (Fig. 2). When adequate decompression of the spinal cord has been achieved, the concavity of the compressed spinal cord dura mater is converted to convexity toward the cavity. This endoscopic operation is performed with the assistance of the videomonitor. Operating within the reversed image of a 70š-angled lens endoscope requires practice. Once adequate decompression is achieved, the endoscope can be removed and repeated Valsalva maneuvers performed using saline to fill the surgical space to confirm intact pleura. The trocar is then removed and a couple of stitches are placed at the paraspinal fascia. The surgical wound is infiltrated with a local anesthetic agent to minimize postoperative incisional pain. Then subcuticular skin closure is made and a small bandage is applied. 


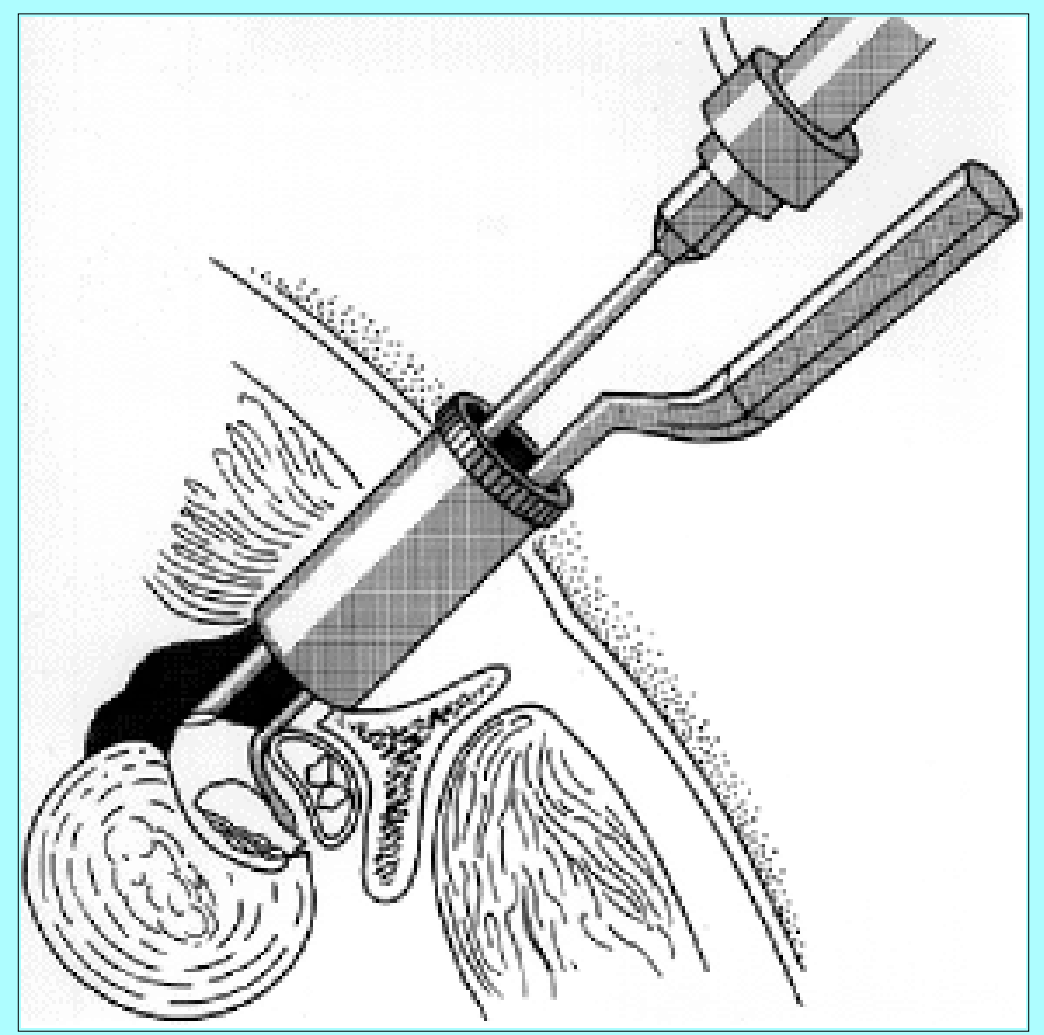

Fig. 2. Schematic drawing showing the 70š-angled endoscope as it is mounted to a custom-made endoscope holder. Under endoscopic visualization, the protruding disc and bone spur compressing the spinal cord medially are pushed away from the spinal cord toward the cavity using a long-armed downward-biting curette and removed using a curved pituitary rongeur.

This endoscopic microscopic thoracic discectomy via a transpedicular approach has been performed on an outpatient basis in patients with radiculopathy. Patients with myelopathy who underwent this procedure were kept in the hospital overnight.

\section{ILLUSTRATIVE CASES}

\section{Case 1}

This 31-year-old woman underwent endoscopic transthoracic discectomy at T9-10, performed by another surgeon, for right-sided lower thoracic radicular pain in February 1995. She experienced improvement in her pain postoperatively for a few months and then noted gradual recurrence of right-sided lower thoracic radicular pain. Over the next 6 months her symptoms progressively worsened with a new development of right leg spastic weakness and urinary urgency, frequency, and incontinence.

Examination. Neurological examination revealed hyperreflexia of both lower extremities, which was worse on the right side than on the left, and a few beats of right ankle clonus. Magnetic resonance (MR) images of the thoracic spine revealed a right-sided disc protrusion that compressed the spinal cord at T9-10 (Fig. 3). 


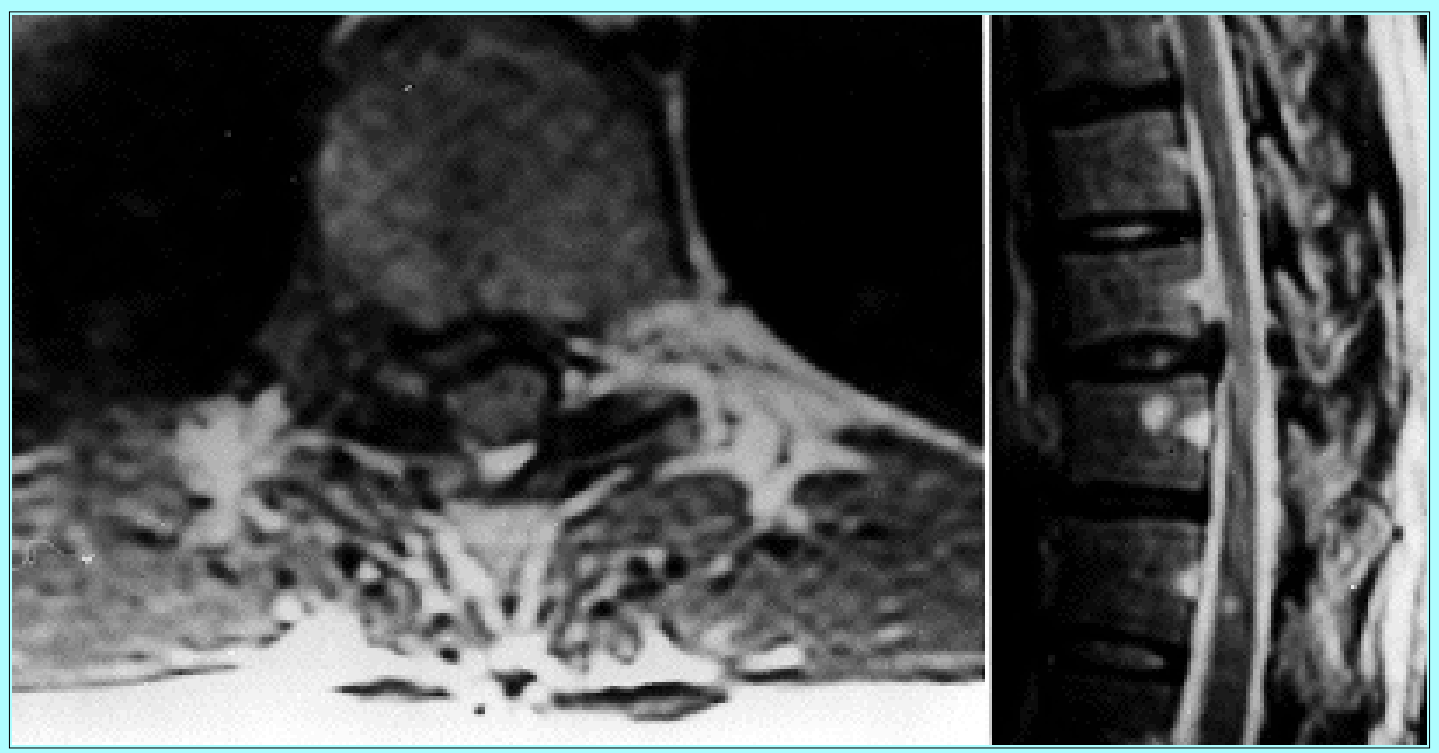

Fig. 3. Case 1. Axial (left) and sagittal (right) MR images of the thoracic spine revealing disc protrusion at T9-10 that pushes the spinal cord posterolaterally to the right.

Operation. The patient underwent an endoscopic microscopic T9-10 discectomy via a right-sided transpedicular approach in May 1996 (Fig. 4). She stayed in the hospital overnight postoperatively.

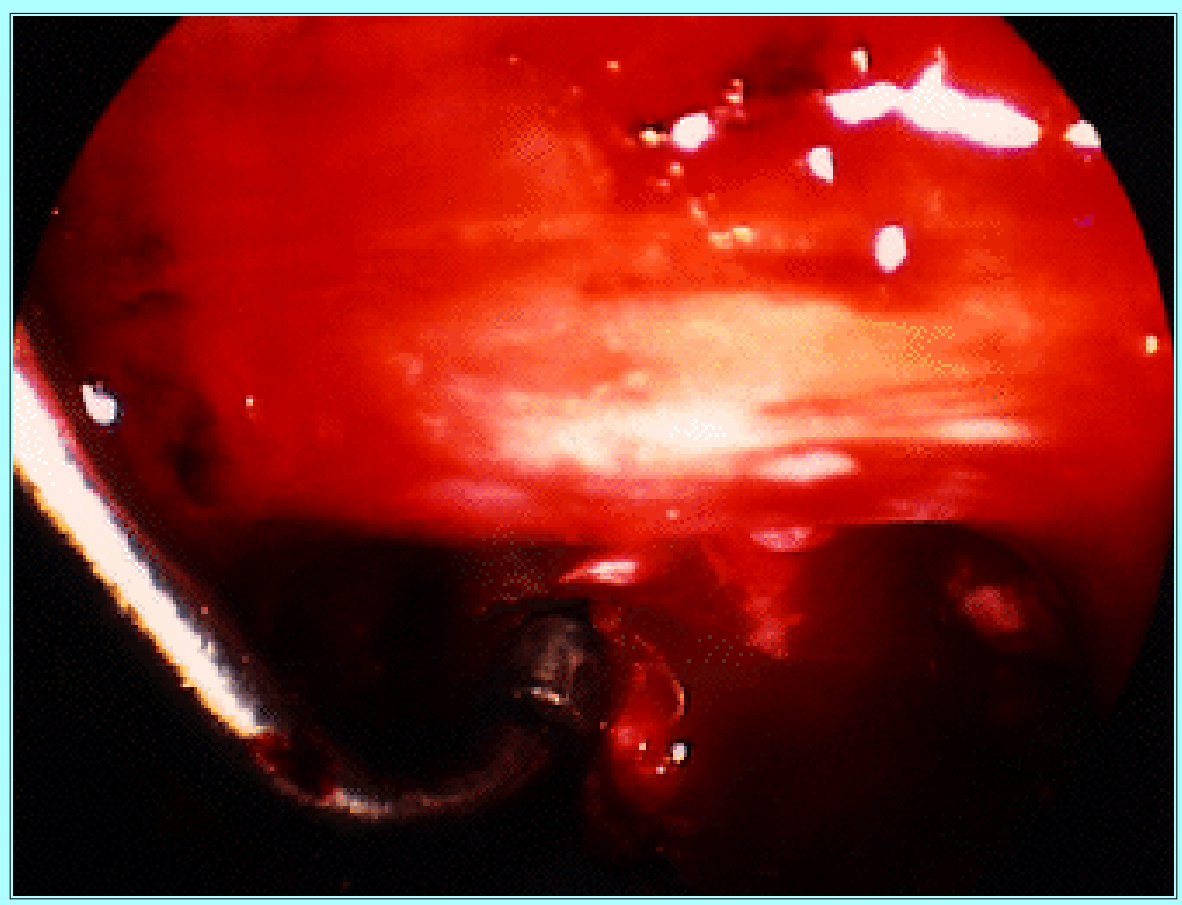

Fig. 4. Case 1. Endoscopic view of the ventral aspect of the spinal cord dura mater. Surgical instruments are gently curved at almost a 90 š angle as they extend beneath the 70š-angled endoscope to reach the contralateral side of the intervertebral space ventrally to the spinal cord.

Postoperative Course. The patient showed complete recovery from myelopathy and resolution of her right-sided lower thoracic radicular pain at 6 weeks postoperatively. Postoperative MR images of her thoracic spine demonstrated complete decompression of the spinal cord (Fig. 5). 


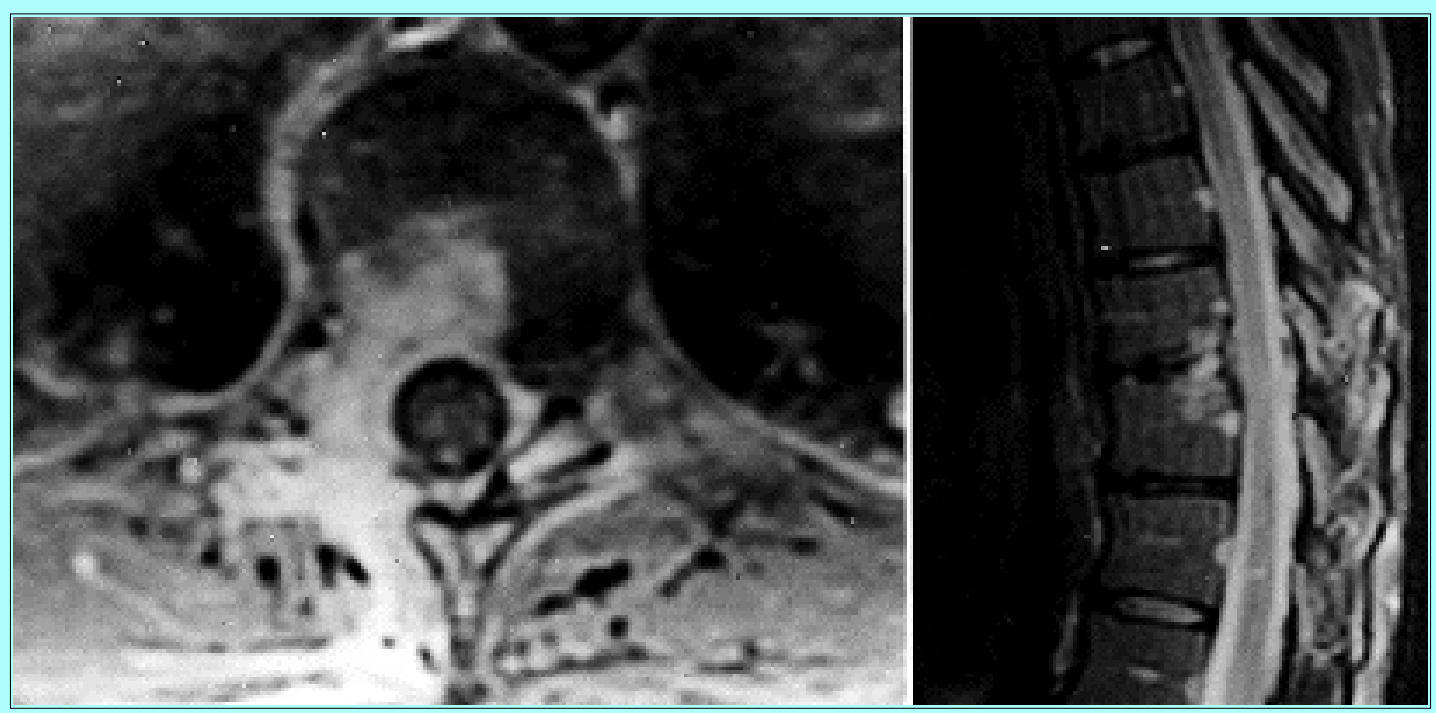

Fig. 5. Case 1. Axial (left) and sagittal (right) postoperative MR images of the thoracic spine demonstrating complete decompression of the spinal cord.

\section{Case 2}

This 52-year-old woman was referred to the University of Pittsburgh Medical Center for right-sided lower thoracic and abdominal pain, which had developed 10 months earlier when she was involved in a motor vehicle accident. She had undergone a T7-8 discectomy via a right-sided costotransversectomy approach performed by another surgeon at a different institution 3 months earlier, without relief of her pain.

Examination. The patient's neurological examination was normal. An MR image of her thoracic spine showed right posterolateral thoracic disc protrusion at T10-11 in addition to residual disc or postoperative changes at T7-8. The distribution of the patient's pain, which was located at her right-sided posterior lower thoracic region and radiated toward her right-sided lower abdomen, strongly suggested that the T10-11 disc protrusion was at fault. Pre- and postoperatively, the patient had been treated conservatively with physical therapy and medications that gave her no pain relief.

Operation. In April 1996, the patient underwent an endoscopic microscopic transpedicular discectomy at T10-11 via a right-sided approach. The operation was uneventful.

Postoperative Course. The patient's radicular pain was relieved immediately after the operation and she was discharged home on the same day. A follow-up visit 6 weeks later confirmed complete relief of her radicular pain. Postoperative MR imaging demonstrated complete decompression of the T10-11 disc protrusion.

\section{DISCUSSION}

In 1978 Patterson and Arbit[5] introduced a transpedicular approach for thoracic discectomy. Stillerman, et al.,[8] recently reported a transfacet approach as access for thoracic discectomy. They performed medial facetectomy via a 4-cm-long skin incision. The surgical technique described here is very similar to, albeit somewhat modified from, those techniques. The intervertebral disc in the thoracic spine is very close to the upper portion of the pedicle of the lower vertebra. When the pedicle is completely preserved as described by Stillerman, et al., the vertical extent of surgical exposure is somewhat limited for removal of the osteophyte at the superior portion of the caudal vertebra. Most thoracic disc protrusion is 
a combination of spondylotic spur and intervertebral disc herniation, which is often calcified. In my technique, only the upper portion of the pedicle of the caudal vertebra, which is approximately 2 to $3 \mathrm{~mm}$ in diameter, is removed using a high-speed drill. As Patterson and Arbit reported, it is important to create a cavity lateral to the spinal cord first, by removing both the bone spur of the rostral and caudal vertebra and the intervertebral disc before any attempt at removing the disc medially.

It can be cumbersome to accommodate various heavy surgical equipment in the operating room. I originally attempted to perform the entire procedure with the endoscope using 0-, 30-, and 70š-angled lenses to eliminate the use of the operating microscope. However, the clarity and stereoscopic perception of the surgical field using the operating microscope is still quite superior to that of the endoscopic view on the monitor. One may maintain better control while using a high-speed drill and other microsurgical instruments in conjunction with the operating microscope than with the endoscope. Use of an endoscope holder is essential to ensure a steady image on the monitor and it also allows a surgeon to use both hands freely. An endoscope cleaning device is another integral element in this endoscopic technique; it permits a surgeon to operate continuously without interruptions caused by removal of a foggy or blood-stained lens for cleaning.

Endoscopic transthoracic discectomy was introduced recently.[7] That transthoracic endoscopic technique still requires three or four separate skin incisions and postoperative chest tube drainage. Although other surgeons have mentioned occasional use of endoscopes to inspect the ventral aspect of the spinal cord dura mater during a posterior approach to thoracic disc protrusions, this is the first report of an actual operation performed with the aid of an endoscope via a posterior approach.[4,8] Operating with the 70š-angled lens endoscope requires a good deal of practice to become familiar with the reversed perception of the surgical field. This surgical technique has been used in eight patients; it has been performed on an outpatient basis for patients with radiculopathy and only required an overnight stay for patients with myelopathy.

\section{Acknowledgments}

The author wishes to thank Arthur P. Nestler, B.S.N., and Robin Coret, B.A., for their assistance with the preparation of this manuscript.

\section{References}

1. Arseni C, Nash F: Thoracic intervertebral disc protrusion. A clinical study. J Neurosurg 17:418-430, 1960

2. Hulme A: The surgical approach to thoracic intervertebral disc protrusions. J Neurol Neurosurg Psychiatry 23:133-137, 1960

3. Larson SJ, Holst RA, Hemmy DC, et al: Lateral extracavitary approach to traumatic lesions of the thoracic and lumbar spine. J Neurosurg 45:628-637, 1976

4. Le Roux PD, Haglund MM, Harris AB, et al: Thoracic disc disease: experience with the transpedicular approach in twenty consecutive patients. Neurosurgery 33:58-66, 1993

5. Patterson RH Jr, Arbit E: A surgical approach through the pedicle to protruded thoracic discs. J Neurosurg 48:768-772, 1978 
6. Perot PL Jr, Munro DD: Transthoracic removal of midline thoracic disc protrusions causing spinal cord compression. J Neurosurg 31:452-458, 1969

7. Rosenthal D, Rosenthal R, de Simone A: Removal of a protruded thoracic disc using microsurgical endoscopy. A new technique. Spine 19:1087-1091, 1994

8. Stillerman CB, Chen TC, Day JD, et al: The transfacet pedicle-sparing approach for thoracic disc removal: cadaveric morphometric analysis and preliminary clinical experience. J Neurosurg 83:971-976, 1995

Manuscript received September 10, 1996.

Accepted in final form February 21, 1997.

Address reprint requests to: Hae-Dong Jho, M.D., Ph.D., Department of Neurological Surgery, Presbyterian University Hospital, Suite B-400, 200 Lothrop Street, Pittsburgh, Pennsylvania 15213.

This article appeared in the July 1997 issue of Journal of Neurosurgery. 